\title{
The new pre-cataclysmic binary PG 2200+085
}

\author{
V. Shimansky ${ }^{1,2,7}$, N. A. Sakhibullin ${ }^{1,2,7}$, I. Bikmaev ${ }^{1,2}$, H. Ritter ${ }^{3}$, V. Suleimanov ${ }^{4,1,7}$, N. Borisov ${ }^{5}$, and A. Galeev ${ }^{1,2,6,7}$ \\ 1 Kazan State University, Kremlevskaja Str., 18, 420008 Kazan, Russia \\ e-mail: Slava.Shimansky@ksu.ru \\ 2 Tatarstan Academy of Science, Baumana Str. 20, 420111 Kazan, Russia \\ 3 Max-Planck-Institut für Astrophysik, Karl-Schwarzschild-Strasse 1, 85740 Garching, Germany \\ 4 Institut für Astronomie und Astrophysik, Universität Tübingen, Sand 1, 72076 Tübingen, Germany \\ 5 Special Astrophysical Observatory, 369167 Nizhnij Arkhyz, Russia \\ ${ }^{6}$ Department of Theoretical Physics, Tatar State Humanitarian Pedagogical University, Tatarstan Str. 2, 420021 Kazan, Russia \\ 7 Kazan Branch of Isaac Newton Institute, Santiago, Chile
}

Received 6 March 2006 / Accepted 24 April 2006

\section{ABSTRACT}

\begin{abstract}
Aims. We present the results of spectroscopic- and orbit-sampled photometric observations of the faint UV-excess object PG 2200+085.

Methods. The optical CCD photometry observations of this object were performed by the Russian-Turkish 1.5-m telescope RTT150 at the TUBITAK National Observatory (Turkey). The long-slit optical spectroscopy observations with $2.6 \AA$ resolution were carried out by 6-m telescope BTA at the Special Astrophysical Observatory (Russia).

Results. The photometric variations over two nights are almost sinusoidal with an amplitude $\Delta m_{V}=0.04$ and a period of $P=0.3186 \mathrm{~d}$. Such a light curve is typical of a detached close binary with an illumination effect or the ellipsoidal deformation of a secondary star. The observed spectrum clearly displays a featureless blue continuum of a hot component and a rich absorption-line and molecular band K-star spectrum. The CaII line profiles with strong emission cores are remarkably similar to those of V471 Tau. Conclusions. We tentatively classify PG $2200+085$ as a pre-cataclysmic binary of the V471 Tau type.
\end{abstract}

Key words. stars: binaries: close - stars: binaries: spectroscopic - stars: individual: PG 2200+085 - stars: novae, cataclysmic variables

\section{Introduction}

PG $2200+085=$ USNO-A2.0 0975-20754051 $\left(\alpha_{2000}=\right.$ $\left.22^{\mathrm{h}} 03^{\mathrm{m}} 19.72, \delta_{2000}=08^{\circ} 45^{\prime} 36^{\prime \prime} .5\right)$ is one of the faint ultraviolet sources, $m_{V}=14 \mathrm{~m} 21$ included in the Palomar-Green (PG) catalogue green et al. (1986). A finding chart for the object is shown in Fig. 1. The spectrum of this star was classified as composite, and its colour index indicates radiation excess in the red spectral region. Later, Schultz et al. (1996) found an absorption feature in the spectrum of PG 2200+085 in the region of the $\mathrm{H}_{\alpha}$ line. Apart from that, PG $2200+085$ has not been studied in any further detail.

During the preliminary photometric observations of PG catalogue objects that we performed in 2002, we found indications of light variations of PG $2200+085$ with an amplitude of $\Delta m_{V}=0$. 02 . Therefore, in 2003, we performed a more focused investigation of PG 2200+085, including longtime photometry with a high-quality CCD-detector over several nights and spectroscopy with the 6-m telescope of the Special Astrophysical Observatory (Russia).

\section{Observations}

\subsection{Photometry}

Photometric observations of PG $2200+085$ were performed on September 18-20 2003, with the 1.5-m Russian-Turkish telescope RTT-150 at the TUBITAK National Observatory (Turkey) on Bakirlitepe Mountain (see Table 1). The thermoelectrically

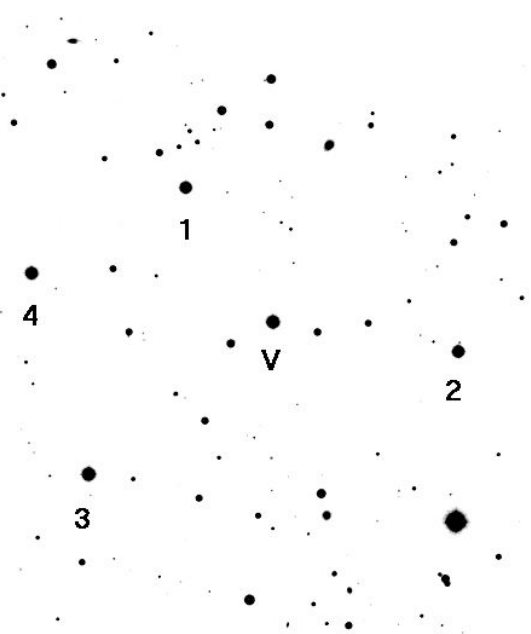

Fig. 1. Finding chart of PG $2200+085$. North is at the top and east to the left. The size is $8^{\prime} \times 8^{\prime}$.

cooled CCD camera ANDOR (model DW436, $2048 \times 2048$ with $13.5 \times 13.5 \mu \mathrm{m}$ pixels) with an operating temperature of $-60{ }^{\circ} \mathrm{C}$ installed at the Cassegrain focus was used. All observations were carried out in the $V$-band with an exposure time of $30 \mathrm{~s}$ and a full frame readout time of $37 \mathrm{~s}$ (binning $2 \times 2$ ). The total observation time during each night was about $6 \mathrm{~h}$ at an average seeing of 2.5 arcsec. Four stars of similar magnitudes in the field of the CCD $\left(8^{\prime} \times 8^{\prime}\right)$, which are identified in Fig. 1, have been used 
Table 1. Log of photometric observations.

\begin{tabular}{cccc}
\hline \hline Data & $\begin{array}{c}\text { Start } \\
\text { JD }\end{array}$ & $\begin{array}{c}\text { Duration } \\
\text { hours }\end{array}$ & $\begin{array}{c}\text { Number } \\
\text { of points }\end{array}$ \\
\hline 19.09 .2003 & 2452901.29470 & 6.25 & 331 \\
20.09 .2003 & 2452902.29269 & 6.16 & 327 \\
\hline
\end{tabular}

Table 2. Variable and comparison stars. Coordinates and magnitudes are from the USNO-2A catalogue (Monet et al. 1998). The stars are also identified in Fig. 1.

\begin{tabular}{lccccc}
\hline \hline Star & $V($ RTT $)$ & $B$ & $V$ & $\alpha_{2000}$ & $\delta_{2000}$ \\
\hline PG 2200 & 14.36 & 14.8 & 14.4 & 220319.7 & +084536 \\
Ref.1 & 14.72 & 14.8 & 14.6 & 220324.4 & +084722 \\
Ref.2 & 14.61 & 15.2 & 14.7 & 220309.2 & +084512 \\
Ref.3 & 14.29 & 14.5 & 14.3 & 220329.3 & +084335 \\
Ref.4 & 14.64 & 15.4 & 14.8 & 220332.6 & +084614 \\
\hline
\end{tabular}

Table 3. $\log$ of spectroscopic observations. $N$ : the spectrum number, $S / N$ : signal-to-noise ratio, $\varphi_{1}$ and $\varphi_{2}$ : orbital phases with respect to ephemeris 1 and 2.

\begin{tabular}{cccccc}
\hline \hline$N$ & UT & HJD & $S / N$ & $\varphi_{1}$ & $\varphi_{2}$ \\
\hline 1 & $22^{\mathrm{h}} 16^{\mathrm{m}}$ & 2452855.4326 & 91 & 0.354 & 0.177 \\
2 & $22^{\mathrm{h}} 26^{\mathrm{m}}$ & 2452855.4395 & 103 & 0.376 & 0.188 \\
3 & $22^{\mathrm{h}} 37^{\mathrm{m}}$ & 2452855.4468 & 98 & 0.398 & 0.199 \\
\hline
\end{tabular}

as comparison stars (see Table 2). Five Landolt standard stars have been observed over both nights, and they have been used to determine the $V$-magnitudes of the reference stars in the field around PG 2200+085. The brightness of these stars was constant within the error of the differential photometry of the two objects $\left(\Delta m_{V}=0.01\right)$.

\subsection{Spectroscopy}

Spectroscopic observations of PG 2200+085 were carried out at the 6-m telescope BTA of the Special Astrophysical Observatory with a long-slit spectrograph Afanasiev et al. (1995) and with a nitrogen-cooled CCD $(1024 \times 1024$ with $24 \times 24 \mu$ m pixels $)$ installed at the prime focus. Observations were performed on August 3, 2003, under excellent weather conditions, with an average seeing of about 1 .'3 (see Table 3). We used a 1302 lines $\mathrm{mm}^{-1}$ grating, which gives a $\Delta \lambda=2.6 \AA$ resolution in the wavelength region $\Delta \lambda 3860-5100 \AA$. Three subsequent spectra with the same exposure time of $600 \mathrm{~s}$ and with a signal-to noise ratio of $S / N \approx 100$ were taken. For the calibration of wavelengths, fluxes, and radial velocities Ar-Ne-He lamp spectra and spectra of the spectrophotometric standard star BD +28 2106 from the review by Bohlin (1996) were taken.

The photometric and spectroscopic data reduction was performed using the MIDAS (Ballester 1992) program package.

\section{Data analysis}

\subsection{The light curves and ephemeris}

The dependence of the magnitude $m_{V}$ of PG $2200+085$ versus the Julian date of our observations on September 19 and 20, is shown in Fig. 2. It is obvious that the light curves during both nights are almost sinusoidal, with a similar amplitude of

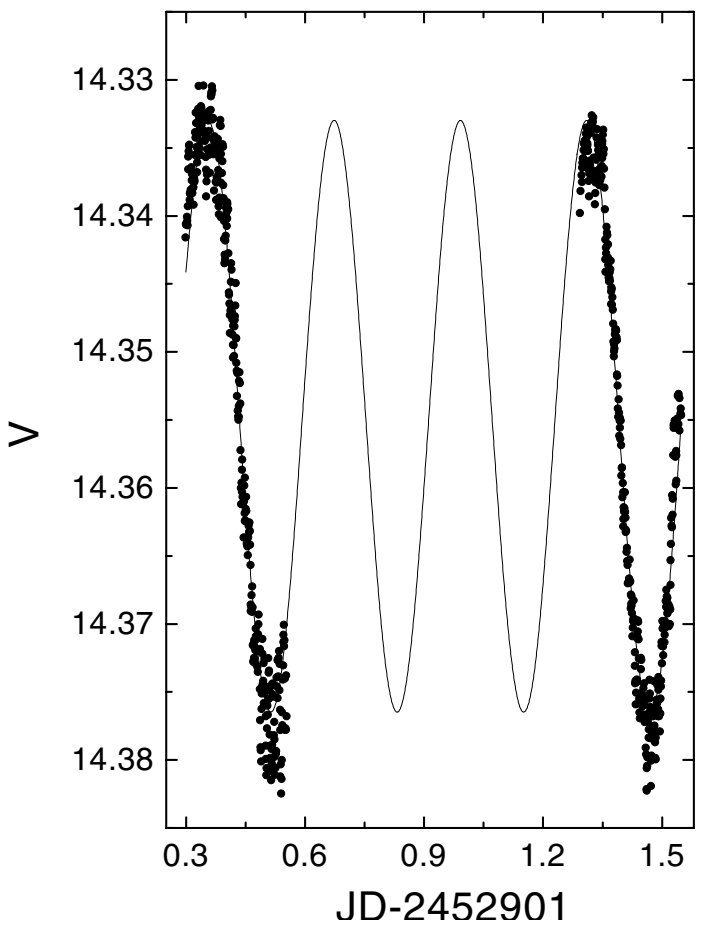

Fig. 2. The light curve of PG $2200+085$ on September 19-20, 2003. The solid curve is the sine function best fitted to the observed data.
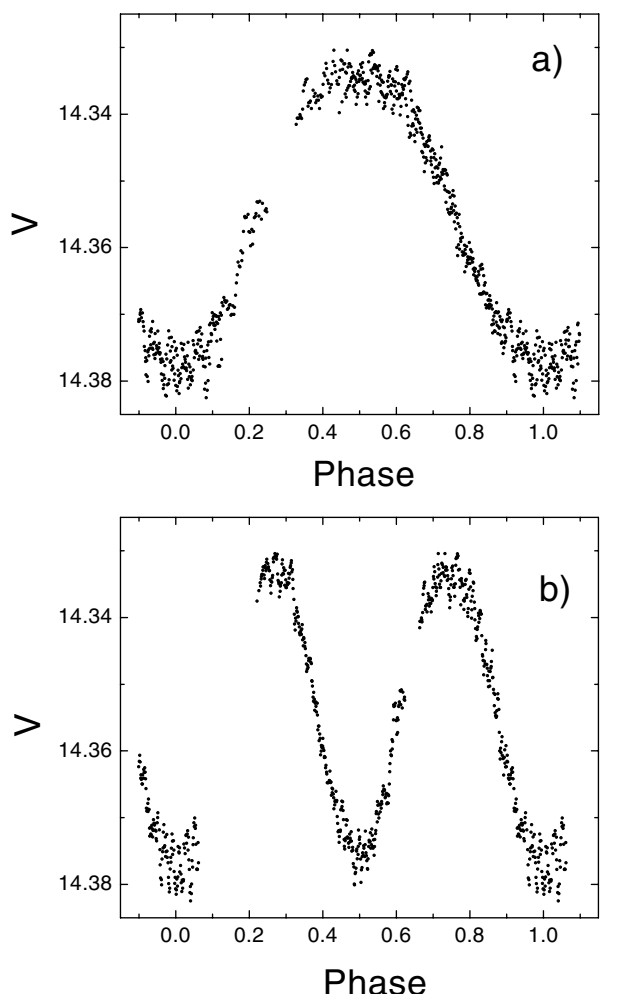

Fig. 3. The light curves of PG $2200+085$ folded with the period 0.31858 a) and 0.63716 b).

$\Delta m_{V}=0$ m 04 . The fitting of the light curve with a simple sine by the $\chi^{2}$ technique resulted in the following ephemeris:

$\mathrm{HJD}=2452900.558( \pm 0.001)+0.31858( \pm 0.00022) \varphi$,

where $\varphi=0.0$ corresponds to the minimum of $m_{V}$. The light curve folded with this period is shown in Fig. 3a. Such a light curve is typical of a close binary with a reflection effect where 
the ultraviolet radiation of the hot primary component is absorbed and reemitted in the optical region from the facing hemisphere of the secondary.

Fitting the light curve by the three-parameter expression

$m_{V}=m_{0}+\frac{m_{1}}{2} * \cos (2 \pi \varphi)+\frac{m_{2}}{2} * \cos (4 \pi \varphi)$

leads to $m_{1}=0$. $045 \pm 0.0003$ and $m_{2} \ll 0$. 002 . The small value of $m_{1}$ means that either the reflection effect or the orbital inclination is small, and the very small value of $m_{2}$ indicates that any superposed ellipsoidal variations in this light curve are very small.

However, based on the available photometric data, we cannot exclude the possiblity that the orbital period is twice the value given in (1) and that the light curve is double-humped. The indirect argument in favour of this possibility is the $10 \%$ difference in the amplitude of the variation of $m_{V}$ during the nights September 19-20. Correspondingly, the light curve could also be described (see Fig. 3b) by the ephemeris:

$\mathrm{HJD}=2452900.558( \pm 0.001)+0.63716( \pm 0.00051) \varphi$.

Fitting the light curve with Eq. (2) yields the parameters $m_{1}=$ $0.004 \pm 0.0003$ and $m_{2}=0.044 \pm 0.0003$. Therefore, if ephemeris (3) applies, then the main light variations (0.044) arise due to ellipsoidal deformation of the secondary.

\subsection{The spectra}

Cross-correlation analysis of the Doppler line shifts in the three spectra of PG $2200+085$ showed the constancy of the radial velocities with a measuring accuracy of $\Delta V=3 \mathrm{~km} \mathrm{~s}^{-1}$ during the $30 \mathrm{~min}$ of observations. At the same time, all important features in these spectra had similar intensity. Therefore, we added the three spectrograms. This yielded the final spectrum with $S / N>170$ shown in Fig. 4a. The presence of strong absorption lines from atoms and ions of heavy elements and weak molecular bands allows us to classify one of the components as a main sequence $\mathrm{K}$-star.

To determine the temperature of this star, we computed synthetic spectra for different values of $T_{\text {eff }}$ and with $\log g=4.5$, $[A]=0.0$ and compared them to the observed spectrum. The computations were performed by means of the program SPECTR (Sakhibullin \& Shimansky 199), whereby we ignored the possible reflection effect in the binary. The observed ratios of the intensities of the metal lines are well modelled (see Fig. 4a) with a temperature $T_{\text {eff }}=4600 \pm 200 \mathrm{~K}$. However, the high level of the observed continuum and the low line intensites indicate the presence of additional flux from the hot component of PG 2200+085. To extract the contribution of this component, we subtracted the theoretical spectrum of the K-star (scaled to the best agreement of observed and theoretical line intensities) from the observed spectrum of PG 2200+085. The residual spectrum shown in Fig. 4a does not show any significant absorption or emission lines. The strong absorption feature near $\Delta \lambda 4750-4800 \AA$ is the result of neglecting the molecular bands in the synthetic K-star spectrum. Therefore, this feature is not part of the spectrum of the hot component. We note that the real flux distribution of the hot component is possibly even steeper than shown in Fig. 4a because we have ignored interstellar absorption. As a result, we can conclude that the observed spectrum of PG $2200+085$ is composite (as mentioned by Green et al. 1986), with contributions of a hot white dwarf and a K-star.

At the time of our observations, the radial velocity of the $\mathrm{K}$-star was $V_{\mathrm{r}}=57 \pm 3 \mathrm{~km} \mathrm{~s}^{-1}$. According to our ephemeris (1),
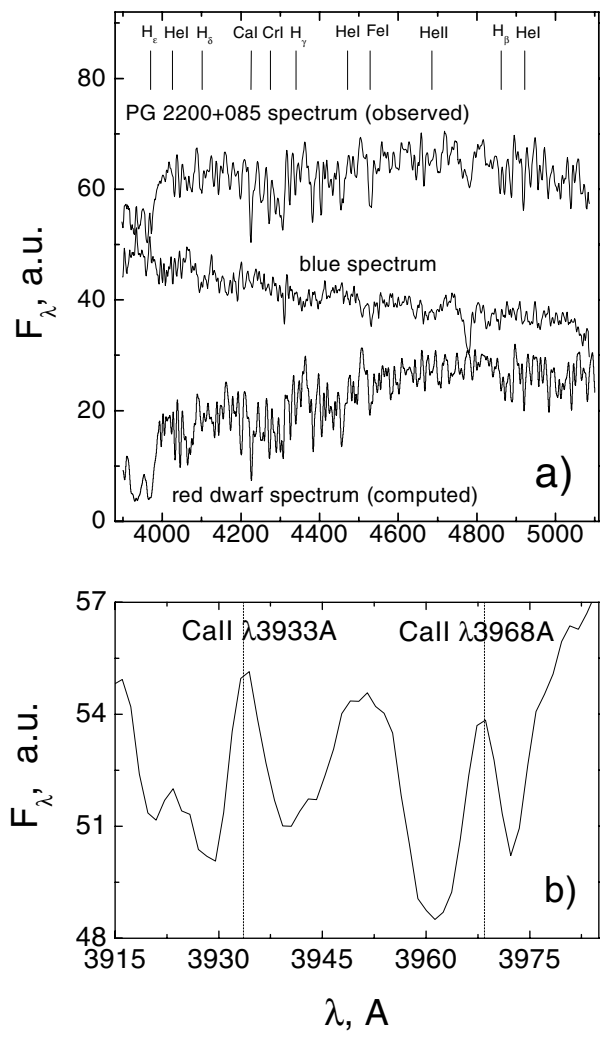

Fig. 4. Observed spectrum of PG 2200+085, theoretical spectrum of the $\mathrm{K}$-star and blue continuum of the hot component a); observed profiles of CaII $\lambda \lambda 3933,3968 \AA$ lines b).

the spectrograms were taken at the phases $\varphi=0.34,0.36$ and 0.38 . The absence of radial velocity variations of the K-star between these phases allows us to set an upper limit on the orbital radial velocity amplitude of $V_{\mathrm{r}} \leq 30 \mathrm{~km} \mathrm{~s}^{-1}$. On the other hand, if we use (3), the corresponding upper limit for the radial velocity amplitude of the K-star is $110 \mathrm{~km} \mathrm{~s}^{-1}$.

In the spectrum of PG $2200+085$ emission lines of ionized calcium $\lambda \lambda$ 3933, $3968 \AA$ can be seen (Fig. 4b). The measured equivalent widths of these lines are $W_{\lambda}(3933)=$ $1.1 \AA$ and $W_{\lambda}(3968)=0.9 \AA$. These widths are too small for a typical K-star without flares. Similar intensities of CaII emission lines are, however, seen in published spectra Skillman \& Patterson (1988) of the pre-cataclysmic binary V471 Tau at an orbital phase $\varphi=0.25$. Moreover, the intensities of all absorption lines in the spectra of V471 Tau and PG 2200+085 are very similar.

\section{Interpretation}

There are a number of possible explanations of the observed basic features. We begin by recalling them:

1. the light curve shown in Fig. 3 is almost sinusoidal with an amplitude of $\Delta V \approx 0$. 045 ;

2. as can be seen from Fig. 4, even in the restricted wavelength range shown the flux from the hot component exceeds the flux of the K-star;

3. the system does not eclipse.

In the following we consider three hypotheses, which could explain the observed light curve.

1. We see a reflection effect in a close detached binary system (a pre-CV). In this case, the orbital period of the system is 
$0.3186 \mathrm{~d}$ and the reflection effect or the orbital inclination is small.

2. We see ellipsoidal variations due to the tidal distortion of the $\mathrm{K}$-star in a close detached binary system (a pre-CV). In this case, the orbital period of the system is $0.6372 \mathrm{~d}$.

3. We see light variations arising from the rotation of a highly magnetized hot white dwarf with an inhomogeneous surface brightness distribution, similar to RE J0317-853 (Barstow et al. 1995b), in a wide binary system. In this case, the period of $0.3186 \mathrm{~d}$ reflects the white dwarf's rotation period.

At present, we do not have sufficient observational data to clearly distinguish between these possibilities. However, there is a statistical argument against the third hypothesis. Hot $\left(T_{\text {eff }}>\right.$ $40000 \mathrm{~K})$ and strongly magnetized white dwarfs, such as RE J0317-853, seem to be very rare objects, much rarer than pre-CVs. Indeed, only four such white dwarfs (Wickramasinghe $\&$ Ferrario 2000) are currently known. In addition, none of them has an unresolved, i.e., very close companion, that can be found by spectroscopic methods only. From this we may conclude that a priori the probability for PG $2200+085$ being a pre-CV is much higher than for a hot and strongly magnetized white dwarf with a K-star companion. Therefore, we consider the first two of the above hypotheses more likely.

Based on the available photometric data alone, and lacking radial velocity data, it is not possible to distinguish between a reflection effect and ellipsoidal variations. Here, however, we wish to discuss arguments that point strongly in favour of the shorter of the two periods, i.e., that the observed brightness variations are due to a reflection effect.

The second observational point allows us to derive a rough lower limit of the primary's effective temperature. Using StefanBoltzmann's law we get

$T_{\text {eff, } 1}>\left(\frac{R_{2}}{R_{1}}\right)^{1 / 2} T_{\text {eff, },}$,

where $R_{1}$ and $R_{2}$ are, respectively, the radius of the primary and the secondary, and $T_{\text {eff, } 2}$ the secondary's effective temperature. Adopting typical values for the white dwarf, i.e., $M_{1}=0.6 M_{\odot}$, $R_{1}=0.01 R_{\odot}$, and $T_{\text {eff }, 2}=4600 \mathrm{~K}$, we obtain a lower limit $T_{\text {eff, } 1}>38500 \mathrm{~K}$. Thus, if the primary is a white dwarf (with a radius $\sim 0.01 R_{\odot}$ ), then it must be rather hot and, therefore, could also account for a reflection effect on the facing hemisphere of the secondary. For our estimate we have assumed that the K-star secondary has a mass of $0.7 M_{\odot}$ and a radius of $0.7 R_{\odot}$. It is clear that the resulting primary temperature is only a lower limit. It is possible to derive an even stronger limit on the temperature of the hot star. Both stars have comparable fluxes at 5000-6000 A. At these wavelengths, the spectral energy distribution of the K-star has its maximum, whereas for the hot star, we are seeing the Rayleigh-Jeans tail of its spectral energy distribution. Assuming black bodies for both stars, we can estimate the temperature of the hot component:

$$
T_{1} \approx 6 \times 10^{5} \mathrm{~K}\left(\frac{\lambda}{6000 \AA}\right)^{4}\left(\frac{T_{2}}{4600 \mathrm{~K}}\right)^{5}\left(\frac{R_{2}}{0.7 R_{\odot}}\right)^{2}\left(\frac{R_{1}}{0.01 R_{\odot}}\right)^{-2}
$$

PG 2200+085 is not known to be a soft X-ray source ${ }^{1}$, therefore the hot component has a temperature less than $\approx 2 \times 10^{5} \mathrm{~K}$ and a radius larger than $\approx 0.02 R_{\odot}$.

We can also make a simple estimate of the amplitude of the resulting reflection effect as follows: assuming that the bolometric luminosity of the facing hemisphere of the secondary is the

\footnotetext{
${ }^{1}$ http://wave. xray.mpe.mpg.de/rosat/catalogue
}

sum of half the secondary's intrinsic luminosity plus the illumination luminosity, i.e.,

$L_{2, \text { front }}=\frac{1}{2} L_{\mathrm{bol}, 2}+\frac{1}{4}\left(\frac{R_{2}}{a}\right)^{2} L_{\mathrm{bol}, 1}$

and that the luminosity of the hemisphere in the shadow of the white dwarf is

$L_{2, \text { back }}=\frac{1}{2} L_{\text {bol, }, 2}$

we obtain from Eqs. (4)-(6)

$\frac{L_{2, \text { front }}}{L_{2, \text { back }}}>1+\frac{1}{2}\left(\frac{R_{2}}{a}\right)^{2}$.

We note that Eq. (7) overestimates the ratio $L_{2 \text {,front }} / L_{2 \text {,back }}$ because we have implicitely assumed parallel illumination rather than illumination from a relatively nearby point source, and because the secondary loses less than half of its luminosity over the illuminated hemisphere. In fact, if the irradiating flux greatly exceeds the secondary's (unperturbed) flux, energy loss through the irradiated hemisphere will be essentially suppressed (see, e.g., Büning \& Ritter 2004) and the first term on the right-hand side of Eq. (7) vanishes.

From our estimate of the K-star parameters, together with $M_{1}=0.6 M_{\odot}$ and the short orbital period $P=0.3186 \mathrm{~d}$, we obtain $\Delta m_{\text {bol }}>0.057 \mathrm{mag}$ as a lower limit of the reflection effect. This is probably big enough to account for the observations. If, on the other hand, $P=0.6372 \mathrm{~d}$, we still get a contribution from the reflection effect of $\Delta m_{\text {bol }}>0.02$ mag. Such a contribution is, however, not seen in the observed light curve. Thus, if $P=0.6372 \mathrm{~d}$, the modulation, being an almost a pure sine at half that period, must be entirely due to ellipsoidal variations with essentially no contribution from a reflection effect. Because the amplitudes of both effects, i.e., reflection effect and ellipsoidal variations, depend in a similar way on the orbital incination (both vanish for very low inclinations), having ellipsoidal varations without a reflection effect means that there is no reflection effect at all.

Although the above estimate of the amplitude of the reflection effect shows that there should be some non-negligible contributions, one could still argue that this estimate is inadequate. Therefore, let us briefly address the possibility of ellipsoidal variations. Now $P=0.64716 \mathrm{~d}$. With the above standard parameters, we find that the radius of the secondary $R_{2}$ in units of its Roche radius $R_{2, \mathrm{R}}$ is $\sim 0.52$. Because the tidal deformation of a star that is only about half the size of its Roche radius is small and because the orbital inclination can also not be too high (note the absence of eclipses and the low limit on the radial velocity amplitude), we would argue that the observed amplitude of the light variations cannot be explained in terms of ellipsoidal variations.

Finally, there is an even more compelling argument in favour of the reflection effect interpretation: in Fig. 5 we show the position of 43 pre-cataclysmic binaries listed in Table 4 in a $T_{\text {eff, } 1}-P$ diagram, in which the size of each symbol (filled circles) indicates the amplitude of the observed reflection effect together with the lowest possible position(s) of PG 2200+085 (open circles), based on our above estimate of the primary's effective temperature and the two possible orbital periods. First, we see the expected trend, namely that the larger the amplitude of the reflection effect, the higher the effective temperature of the irradiating star and the lower the orbital period. Second, we see that based on its position(s) in this diagram, PG $2200+085$ is also expected 
Table 4. Pre-cataclysmic binaries for which the orbital period and the effective temperature $T_{\text {eff, }, 1}$ of the hot component are known. A + in the column giving the amplitude of the reflection effect indicates that a reflection effect of unspecified (and probably small) amplitude has been seen.

\begin{tabular}{|c|c|c|c|c|c|c|c|}
\hline Object & $\begin{array}{l}\text { Orbital } \\
\text { period } \\
\text { (d) }\end{array}$ & $\begin{array}{l}\text { Secondary } \\
\text { spectrum }\end{array}$ & $\begin{array}{l}\text { Primary } \\
\text { spectrum }\end{array}$ & $\begin{array}{c}T_{\mathrm{eff}, 1} \\
\left(10^{3} \mathrm{~K}\right)\end{array}$ & $\begin{array}{c}\text { Reflect. } \\
\text { effect } \\
\text { (mag) }\end{array}$ & Filter & References \\
\hline $1017-0838$ & 0.072994 & & $\mathrm{sdB}$ & $30.3 \pm 0.1$ & 0.083 & V & 1 \\
\hline $0705+6700$ & 0.095647 & M V & $\mathrm{sdB}$ & $28.8 \pm 0.9$ & 0.16 & $V$ & 2 \\
\hline NY Vir & 0.101016 & M V & $\mathrm{sdB}$ & $33 \pm 3$ & 0.20 & $V$ & 3 \\
\hline HR Cam & 0.103063 & M V & DA3 & 19 & 0.03 & $V$ & 4 \\
\hline MT Ser & 0.113227 & & $\mathrm{sdO}$ & $50 \pm 5$ & 0.3 & $B$ & 5,6 \\
\hline HW Vir & 0.116720 & & $\mathrm{sdB}$ & $28.5 \pm 0.2$ & 0.21 & $V$ & 7,8 \\
\hline $2237+8154$ & 0.123681 & M3-4 V & DA & $11.5 \pm 0.5$ & & & 9 \\
\hline NN Ser & 0.130080 & M6.5 V & DAO1 & $55 \pm 8$ & 0.6 & $V$ & 10,11 \\
\hline $1347-1258$ & 0.150758 & M4e V & $\mathrm{DA}$ & $14.1 \pm 0.1$ & & & 12,13 \\
\hline $1150+5956$ & $0.1523:$ & & & $111 \pm 10$ & & & 14,15 \\
\hline $\mathrm{J} 1129+6637$ & 0.171 & $\mathrm{M} 4.5 \mathrm{~V}$ & & $17 \pm 2$ & & & 16 \\
\hline $2333+3927$ & 0.171802 & M3-4 V & $\mathrm{sdB}$ & $37.6 \pm 1.0$ & 0.31 & $V$ & 17 \\
\hline MS Peg & 0.173666 & M3-5 V & DA2 & $22.2 \pm 0.1$ & 0.105 & $V$ & 18,19 \\
\hline BPM 71214 & 0.201626 & $\mathrm{M} 2.5 \mathrm{~V}$ & $\mathrm{DA}$ & $17.2 \pm 1.0$ & & & 12 \\
\hline LM Com & 0.258687 & $\mathrm{M} 4+\mathrm{V}$ & DA & 29.3 & 0.14 & $V$ & 19,20 \\
\hline AA Dor & 0.261582 & & $\mathrm{sdO}$ & $42 \pm 1$ & 0.06 & $V$ & 21,22 \\
\hline $2154+4080$ & 0.26772 & M V & DA2 & 30 & 0.16 & $V$ & 23 \\
\hline CC Cet & 0.286654 & M5e V & DA2 & $26.2 \pm 2.0$ & 0.08 & $R$ & 24,25 \\
\hline RR Cae & 0.303700 & M5-6 V & DAwk & 7 & & & 26,27 \\
\hline TW Crv & 0.32762 & M V & $\mathrm{sdO}$ & $105 \pm 20$ & 0.846 & $V$ & 28,29 \\
\hline $1042-6902$ & 0.336784 & $\mathrm{Me} \mathrm{V}$ & DA3 & $20.6 \pm 2.0$ & 0.011 & $R$ & 30,31 \\
\hline GK Vir & 0.344331 & M3-5 V & $\mathrm{DAO}$ & $48.8 \pm 1.2$ & $<0.05$ & $B$ & 32 \\
\hline KV Vel & 0.357113 & & $\mathrm{sdO}$ & $77 \pm 3$ & 0.55 & $V$ & 33 \\
\hline UU Sge & 0.465069 & & $\mathrm{sdO}$ & $87 \pm 13$ & 0.36 & $V$ & 34,35 \\
\hline V477 Lyr & 0.471729 & & $\mathrm{sdO}$ & $60 \pm 10$ & 0.62 & $V$ & 36 \\
\hline $\mathrm{J} 2131+4710$ & 0.521035625 & M4 V & DA2 & $18.0 \pm 1.0$ & & & 37 \\
\hline V471 Tau & 0.521183 & $\mathrm{~K} 2 \mathrm{~V}$ & DA2 & $34.5 \pm 1.0$ & & & 38 \\
\hline HZ 9 & 0.56433 & M5e V & DA2 & 17.4 & & & 39,30 \\
\hline V664 Cas & 0.581648 & G8-K0 V & $\mathrm{sdO}$ & $83 \pm 6$ & 1.15 & $V$ & 41,29 \\
\hline UZ Sex & 0.597259 & M4e V & DA3 & $17.6 \pm 2.0$ & 0.012 & $R$ & 24,42 \\
\hline EG UMa & 0.667579 & M4-5 V & DA4 & $13.1 \pm 0.1$ & & & 43,44 \\
\hline VW Pyx & 0.6758 & & $\mathrm{sdO}$ & $85 \pm 6$ & 1.36 & $V$ & 45,46 \\
\hline $\mathrm{J} 2013+4002$ & 0.70552 & M3-4 V & DAO & $49.0 \pm 0.7$ & + & & 47,48 \\
\hline $2009+6220$ & 0.741226 & M5-6 V & $\mathrm{DA} 2$ & 25 & & & 31 \\
\hline J1016-0520 & 0.78928 & M0-4 V & DAO & $55 \pm 1$ & + & & 48,49 \\
\hline $1136+6646$ & 0.83607 & $\mathrm{~K} 4-7 \mathrm{~V}$ & DAO & 70 & 0.24 & $V$ & 50 \\
\hline Abell 65 & 1.00 & & sd? & 80 & $>0.5$ & $V$ & 51,52 \\
\hline IN CMa & 1.262396 & M0-2 V & DAO & $53 \pm 1$ & 0.075 & $V$ & $12,48,53$ \\
\hline BE UMa & 2.291166 & K3-4 V & DAO & $105 \pm 5$ & 1.3 & $V$ & $54,55,56$ \\
\hline Feige 24 & 4.23160 & M1-2 V & DAO & $56 \pm 1$ & & & 57,58 \\
\hline FF Aqr & 9.20803 & G8 III & sdOB & 42 & 0.22 & $U$ & 58 \\
\hline V651 Mon & 15.991 & A5 V & & 100 & & & 60 \\
\hline IK Peg & 21.7217 & A8 V & DA1 & $35.4 \pm 0.2$ & & & 61 \\
\hline
\end{tabular}

References: 1: Maxted et al. (2002), 2: Drechsel et al. (2001), 3: Killkenny et al. (1998), 4: Maxted et al. (1998), 5: Green et al. (1984), 6: Bruch et al. (2001), 7: Wood et al. (1999), 8: Ibanoglu et al. (2004), 9: Gänsicke et al. (2004), 10: Catalan et al. (1994), 11: Pigulski et al. (2002), 12: Kawka et al. (2002), 13: O'Donoghue et al. (2003), 14: Jacoby et al. (2002), 15: Tovmassian et al. (2004), 16: Raymond et al. (2003), 17: Heber et al. (2004), 18: Schmidt et al. (1995), 19: Shimansky et al. (2003), 20: Orosz et al. (1999), 21: Rauch (2000), 22: Hilditch et al. (2003), 23: Hillwig et al. (1996), 24: Saffer et al. (1993), 25: Somers et al. (1996), 26: Bragaglia et al. (1995), 27: Bruch et al. (2001), 28: Chen et al. (1995), 29: Exter et al. (2003), 30: Kawka et al. (2000), 31: Morales-Rueda et al. (2005), 32: Fulbright et al. (1993), 33: Hilditch et al. (2003), 34: Pollacco et al. (1993), 35: Bell et al. (1994), 36: Pollacco et al. (1994), 37: Maxted et al. (2005) 38: O’Brien et al. (2001), 39: Lanning et al. (1981), 40: Schreiber et al. (2003), 41: Shimanskii et al. (2004), 42: Bruch et al. (1999), 43: Bleach et al. (2000), 44: Bleach et al. (2002), 45: Kohoutek et al. (1982), 46: Exter et al. (2003), 47: Thorstensen et al. (1994), 48: Vennes et al. (1999), 49: Thorstensen et al. (1996), 50: Sing et al. (2004), 51: Bond et al. (1990), 52: Walsh et al. (1996), 53: Barstow et al. (1995a), 54: Wood et al. (1995), 55: Ferguson et al. (1999), 56: Raguzova et al. (2003), 57: Vennes et al. (1998), 58: Vennes et al. (2000), 59: Vaccaro et al. (2003), 60: Mendez et al. (1985), 61: Vennes et al. (1998).

to show a (weak) reflection effect, even for the longer orbital period (in agreement with our rough estimate above). The outliers in this diagram that seem to violate the trend are the systems
FF Aqr and 1150+5956. The contradiction is, however, only apparent. In the case of FF Aqr, the primary is not only a rather hot sdO star, but it also has a much larger radius $\left(\sim 0.16 R_{\odot}\right.$, 


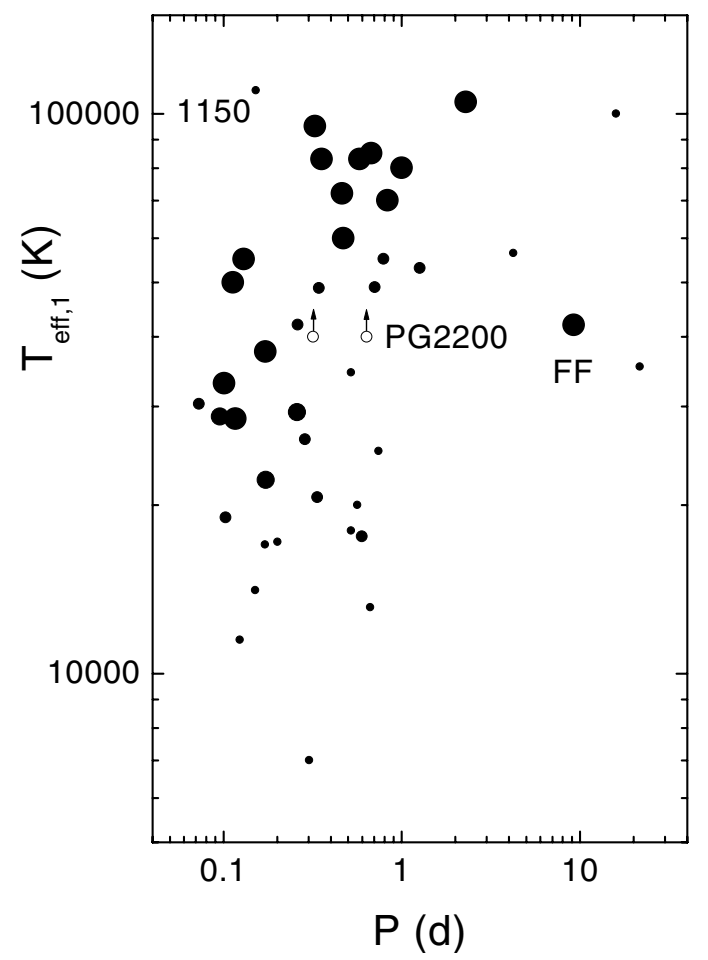

Fig. 5. Effective temperature $T_{\text {eff, }, 1}$ plotted versus orbital period for the systems listed in Table 4 . The size of the filled circles indicates the amplitude of the reflection effect. The smallest symbol is for systems without a reflection effect, the largest for those where $\Delta m_{\text {refl }}>0.2 \mathrm{mag}$, the second to largest for those where $0.2 \mathrm{mag}>\Delta m_{\mathrm{refl}}>0.1 \mathrm{mag}$, and the second to smallest for those where $0.1 \mathrm{mag}>\Delta m_{\mathrm{refl}}>0 \mathrm{mag}$. The lowest position of PG $2200+085$ in this diagram is shown by the two open circles. The labels FF and 1150 refer, respectively, to the systems FF Aqr and 1150+5956.

see Vaccaro \& Wilson 2003) and luminosity than an ordinary white dwarf. This can fully account for the large reflection effect, even at that long orbital period, as has been shown by Vaccaro $\&$ Wilson 2003. In the case of $1150+5956$, the orbital period is a very preliminary and detailed time-resolved photometry which could reveal a reflection effect that is currently unavailable.

From these arguments, we would then conlude that the true orbital period of PG $2200+085$ is $P=0.3186 \mathrm{~d}$ and that the observed periodic modulation is the result of a reflection effect on the K-star caused by the hot primary white dwarf. The comparatively small amplitude of the reflection effect, in turn, results from the relatively low inclination of the system.

\section{Conclusions}

The observations presented in this paper allow us to conclude that PG $2200+085$ is a pre-cataclysmic binary consisting of a hot (pre-) white dwarf and a K-dwarf companion, and with an orbital period of either $P=0.3186 \mathrm{~d}$ or $P=$ $0.6372 \mathrm{~d}$. When comparing PG $2200+085$ to the pre-cataclysmic binaries listed by Schreiber \& Gänsicke (2003), or to the most recent version (release 7.4) of the Ritter \& Kolb (2003) catalogue, we note that PG $2200+085$ is only the second such system consisting of a white dwarf and a K-star companion (the other one being V471 Tau). All other known short-period pre-cataclysmic binaries either consist of a white dwarf and an M-dwarf companion, or, if the companion is a K-star, the hot component is an extremely hot sdO star. From the evolutionary point of view, systems like V471 Tau and PG 2200+085 are interesting because they are the direct progenitors of cataclysmic variables with a comparatively long orbital period, i.e., $P \geq 6 \mathrm{~h}$. We cannot exclude the other possibility, namely, that this object is a wide binary consisting of a hot, highly magnetized white dwarf and a K-star companion. But, according to the statistical arguments presented above, this is much less probable. Nonetheless, if it were true, PG $2200+085$ is an even more interesting object than a pre-CV. For this reason alone, PG $2200+085$ deserves further and detailed study.

In the framework of the pre-CV hypothesis, we have presented theoretical and observational arguments that resolve the orbital period ambiguity in favour of the shorter period $P=$ $0.3186 \mathrm{~d}$, i.e., we favour the interpretation that the optical modulation is due to a reflection effect on the facing hemisphere of the K-star, which, in turn, is caused by irradiation from the moderately hot ( $\left.T_{\text {eff, } 1} \geq 38500 \mathrm{~K}\right)$ white dwarf. However, for a final decision about which of the two proposed periods is the correct one, a detailed radial velocity study of PG $2200+085$ is required.

Acknowledgements. The authors are grateful to the Large Telescopes Program Committee for many years of support of our observational programs, and to the referee, Dr. Barstow, for his interesting suggestion that PG $2200+085$ could be a hot and highly magnetized white dwarf. This work was supported by the Russian Foundation of Fundamental Research (grant 05-02-17744) and by the President program for support of the leading science school (grant NSh-784.2006.2). VS thanks DFG for partial financial support (grant We 1312/35-1).

\section{References}

Afanasiev, V. L., Burenkov, A. N., Vlasiouk, V. V., \& Drabek, S. V. 1995, Preprint SAO RAS, $N$ 234, 128 (in Russian)

Ballester, P. 1992, ESO/ST-ECF Data Analysis Workshop, 177

Barstow, M. A., O’Donoghue, D., Kilkenny, D., Burleigh, M. R., \& Fleming, T. A. 1995 a, MNRAS, 273,711

Barstow, M. A., Jordan, S., O’Donoghue, D., et al. 1995b, MNRAS, 277, 971

Bell, S. A., Pollacco, D. L., \& Hilditch, R. W. 1994, MNRAS, 270, 449

Bleach, J. N., Wood, J. H., Catalan, M. S., et al. 2000, MNRAS, 312, 70

Bleach, J. N., Wood, J. H., Smalley, B., \& Catalan, M. S. 2002, MNRAS, 336, 611

Bohlin, R. C. 1996, AJ, 111, 1743

Bond, H. E., \& Livio, M. 1990, ApJ, 355, 568

Bragaglia, A., Renzini, A., \& Bergeron, P. 1995, ApJ, 443, 735

Bruch, A. 1999, AJ, 117, 3031

Bruch, A., \& Diaz, M.P. 1999, A\&A, 351, 573

Bruch, A., Vaz, L. P. R., \& Diaz, M. P. 2001, A\&A, 377, 898

Büning, A., \& Ritter, H. 2004, A\&A, 423, 281

Drechsel, H., Heber, U., Napiwotzki, R., et al. 2001, A\&A, 379, 839

Catalan, M. S., Davey, S. C., Sarna, M. J., Smith, R. C., \& Wood, J. H. 1994, MNRAS, 269, 879

Chen, A., O'Donoghue, D., Stobie, R. S., et al. 1995, MNRAS, 275, 100

Exter, K. M., Pollacco, D. L., \& Bell, S. A. 2003, MNRAS, 341, 1349

Exter, K., Pollacco, D. L., Maxted, P. F. L., Napiwotzki, R., \& Bell, S. A. 2005, MNRAS, 359, 315

Gänsicke, B. T., Araujo-Betancor, S., Hagen, H.-J., et al. 2004, A\&A, 418, 265

Green, R.F., Liebert, J., \& Wesemael, F. 1984, ApJ, 280, 177

Green, R. F., Schmidt, M., \& Liebert, J. 1986, ApJS, 61, 305

Fulbright, M. S., Liebert, J., Bergeron, P., \& Green, R. 1993, ApJ, 406, 240

Ibanoglu, C., Cakirli, O., \& Evren, S. 2004, A\&A, 141, 1043

Jacoby, G. H., Feldmeier, J. J., Claver, C. F., et al. 2002, AJ, 124, 3340

Ferguson, D. H., Liebert, J., Haas, S., Napiwotzki, R., \& James, T. A. 1999, ApJ, 518,866

Heber, U., Drechsel, H., Ostensen, R., et al. 2004, A\&A, 420, 251

Hilditch, R. W., Harries, T. J., \& Hill, G. 1996, MNRAS, 279, 1380

Hilditch, R. W., Kilkenny, D., Lynas-Gray, A. E., \& Hill, G. 2003, MNRAS, 344, 644

Hillwig, T. C., Gale, A. A., Honeycutt, R. K., \& Rengstorf, A. W. 2002, PASP, 114,756

Kawka, A., Vennes, S., Dupuis, J., \& Koch, R. 2000, AJ, 120, 3250

Kawka, A., Vennes, S., Koch, R., \& Williams, A. 2002, AJ, 124, 2853

Killkenny, D., O’Donoghue, D., Koen, C., Lynas-Gray, A. E., \& van Wyk, F. 1998, MNRAS, 296, 329

Kohoutek, L., \& Schnur, G.F.O. 1982, MNRAS, 201, 21 
Lanning, H. H., \& Pesch, P. 1981, ApJ, 244, 280

Maxted, P. F. L., Marsh, T. R., Moran, C., Dhillon, V. S., \& Hilditch, R. W. 1998 MNRAS, 300, 1225

Maxted, P. F. L., Marsh, T. R., Heber, U., et al. 2002, MNRAS, 333, 231

Maxted, P. F. L., Marsh, T. R., Morales Rueda, L., et al. 2005, MNRAS, 355, 1143

Morales-Rueda, L., Marsh, T. R., Maxted, P. F. L., et al. 2005, MNRAS, 359, 648

Mendez, R. H., Marino, B. F., Claria, J. J., \& van Driel, W. 1985, Rev. Mex. Astron. Astrof., 10. 187

Monet, D., Bird, A., Canzian, G, et al. 1998, VizieR On-line Data Catalog: I/252

O’Brien, M. S., Bond, H. E., \& Sion, E. M. 2001, ApJ, 563, 971

O'Donoghue, D., Koen, C., Kilkenny, D., et al. 2003, MNRAS, 345, 506

Orosz, J. A., Wade, R. A., Harlow, J. J. B., et al. 1999, AJ, 117, 1598

Pollacco, D. L., \& Bell, S. A. 1993, MNRAS, 262, 377

Pollacco, D. L., \& Bell, S. A. 1994, MNRAS, 267, 452

Pigulski, A., \& Michalska, G. 2002, IBVS, 5218

Raguzova, N. V., Shugarov, S. Yu., \& Ketsaris, N. A. 2003, Astron. Rep., 47, 492 Rauch, T. 2000, A\&A, 356, 665

Raymond, S. N., Szkody, P., Hawley, S. L., et al. 2003, AJ, 125, 2621

Ritter, H., \& Kolb, U. 2003, A\&AS, 404, 301

Saffer, R. A., Wade, R. A., Liebert, J., et al. 1993, AJ, 105, 1945

Sakhibullin, N. A., \& Shimanskiy, V. V. 1997, Astr. Rep., 41, 378
Schmidt, G. D., Smith, P. S., Harvey, D. A., \& Grauer, A. D. 1995, AJ, 110, 398 Schreiber, M. R., \& Gänsicke, B. T. 2003, A\&A, 406, 305

Shimansky, V. V., Borisov, N. V., \& Shimanskaya, N. N. 2003, Astron. Rep., 47, 763

Shimansky, V. V., Borisov, N. V., Sakhibullin, N. A., \& Surkov, A. E. 2004, Astron. Rep., 48, 563

Schultz, G., Zuckerman, B., \& Becklin, E. E. 1996, ApJ, 460, 402

Sing, D. K., Holberg, J. B., Burleigh, M. R., et al. 2004, AJ, 127, 2936

Skillman, D. R., \& Patterson, J. 1988, AJ, 96, 976

Somers, M. W., Lockley, J. J., Naylor, T., \& Wood, J. H. 1996, MNRAS, 280, 1277

Thorstensen, J. R., Vennes, S., \& Shambrook, A. 1994, AJ, 108, 1924

Thorstensen, J. R., Vennes, S., \& Bowyer, S. 1996, ApJ, 457, 390

Tovmassian, G. H., Napiwotzki, R., Richer, M. G., et al. 2004, ApJ, 616, 485

Vaccaro, T. R., \& Wilson, R. E. 2003, MNRAS, 342, 564

Vennes, S., \& Thorstensen, J. R. 1994, AJ, 108, 1881

Vennes, S., Christian, D. J., \& Thorstensen, J. R. 1998, ApJ, 502, 763

Vennes, S., Thorstensen, J.R., \& Polomski, E.F. 1999, ApJ, 523, 386

Vennes, S., Polomski, E. F., Lanz, T., et al. 2000, ApJ, 544, 423

Walsh, J. R., \& Walton, N. A. 1996, A\&A, 315, 253

Wickramasinghe, D. T., \& Ferrario, L. 2000, PASP, 112, 873

Wood, J. H., \& Saffer, R. 1999, MNRAS, 305, 820

Wood, J. H., Robinson, E. L., \& Zhang, E.-H. 1995, MNRAS, 277, 87 